\section{P2-226 FISH, VEGETABLE, AND FRUIT INTAKES AND MORTALITY FROM PULMONARY EMBOLISM AMONG JAPANESE MEN AND WOMEN: THE JACC (JAPAN COLLABORATIVE COHORT STUDY FOR EVALUATION OF CANCER RISK) STUDY}

doi:10.1136/jech.2011.142976j.59

${ }^{1} \mathrm{~T}$ Ohira, ${ }^{*} \mathrm{H}$ Iso, ${ }^{2} \mathrm{~K}$ Yamagishi, ${ }^{3} \mathrm{~A}$ Tamakoshi. ${ }^{1}$ Osaka University, Suita, Osaka, Japan; ${ }^{2}$ University of Tsukuba, Tsukuba, Ibaraki, Japan; ${ }^{3}$ Aichi Medical University, Nagakute, Aichi, Japan

Introduction Numerous studies have reported associations of cardiovascular risk factors with pulmonary embolism (PE), but an association of dietary factors with the risk of PE is not fully established. Methods Using a prospective design, we studied the 14.7-year risk of $\mathrm{PE}$ death in relation to dietary factors, such as fish, vegetable, and fruit intakes, in 91280 men and women aged 40-79 years in Japan. The HRs of PE death and 95\% CIs were calculated by using the Cox proportional hazards model.

Results We documented 56 deaths due to PE during 14.7 years Mean values of age were significantly higher among participants with PE than those without PE, but there were no significant differences in body mass index (BMI), hypertension, or diabetes mellitus between participants with PE and those without PE. The age- and sex-adjusted HRs of PE were lower across frequency of fish intake. Compared with the participants in the lowest fish intake group ( $<1$ time/month), the HRs (CIs) of PE for those in the other groups were 0.31 ( 0.09 to 1.07$)$ for $1-2$ times/month, 0.22 (0.08 to $0.60)$ for $1-2$ times/week, 0.19 (0.07 to 0.51$)$ for 3-4 times/week, and 0.18 (0.07 to 0.52 ) for every day ( $\mathrm{p}$ for trend $<0.001)$. The HRs were unchanged by additional adjustment for other PE risk factors. There were no significant associations of vegetable and fruit intakes with the risk of PE death.

Conclusion A greater fish intake is associated with a lower risk of PE death among Japanese men and women.

\section{P2-227 IS COPD A BLESSING IN DISGUISE FOR LUNG CANCER PATIENTS?}

doi:10.1136/jech.2011.142976j.60

\footnotetext{
1,20 Olajide, * 'S Callaghan, ${ }^{2} \mathrm{G}$ Gee. 'Liverpool Primary Care Trust, Liverpool, UK; ${ }^{2}$ Liverpool John Moores University, Liverpool, UK
}

Introduction Although lung cancer is the most common cancer in the world with an estimated 1.61 million new cases diagnosed in 2008 (Cancer Research UK, 2010) little is known about the effect long term lung disease has on the survival rate of lung cancer.

Methods The aim of the study is to compare the 3-year survival rates of lung cancer patient with pre-existing COPD with the 3-year survival rates of patients with lung cancer only. Data from Hospital Episodes Statistics was identified for lung cancer patients as well as for COPD patients.

Results Three year data obtained from Liverpool PCT showed 434 new cases of lung cancer and 48 (11\%) of these new cases are known COPD patients. Inference from the analysed data proved to be contrary to previous knowledge that co-morbidities reduced survival rates of lung cancer patients. Lung cancer patients with COPD showed better survival than lung cancer patients without. This difference is more evident after controlling for age. Lung cancer patients (aged 65 or less) with COPD were seen to have survived more $\left(\operatorname{Pr}>\chi^{2}=0.0223\right)$ than their non-COPD counterparts of the same age category.

Conclusion This study was able to show a difference in the survival rate of the two lung cancer groups researched (those with or without COPD) with patients with COPD surviving more than those without. This difference becomes more significant in patients less than 65 years.

\section{P2-228 DEPRIVATION AND OBESITY-RELATED CHRONIC DISEASES A PROBIT REGRESSION ANALYSIS USING LINKED HOSPITALISATION DATA}

doi:10.1136/jech.2011.142976j.61

D Olajide, ${ }^{*}$ A Avenell. University of Aberdeen, Aberdeen, Scotland, UK

Introduction Chronic diseases associated with obesity reduce quality of life, are a major cause of death, and have a direct impact on the demand for hospital care and the attendant costs. Lifestyles such as diet and physical activity feature prominently among the factors influencing these diseases. However, environmental factors such as deprivation may limit the range of options for healthier lifestyles. We examine factors influencing the risk of obesity-related diseases, and how these differ by degree of deprivation.

Methods We carry out a probit regression analysis of linked hospitalisation episode data from the Scottish Morbidity Records that have been administratively linked to respondents to the Scottish Health Surveys. The outcome measures are obesity-related cancers, heart diseases, stroke, type 2 diabetes, and peripheral vascular diseases identified by International Classification of Diseases (ICD9 and ICD10) codes.

Results Compared with the respective reference groups, men, older age, current smoker, being overweight or obese, and the poorer in general health are associated with a greater risk of an obesity-related disease. On the other hand, being married, education, spending more time doing a sporting activity, and better dietary behaviour are associated with a lower risk of an obesity-related disease. However, increasing time spent doing sporting activity reduces the risk of an obesity-related disease in the most deprived group, while a better dietary behaviour achieves this in the least deprived group.

Conclusion Interventions targeting lifestyles to reduce obesityrelated morbidity in the population may have differential effects between the least and the most disadvantaged groups.

\section{P2-229 NON-ALCOHOLIC FATTY LIVER DISEASE: PREVALENCE AND TRANSITION PROBABILITY IN A RANDOM SAMPLE POPULATION-BASED STUDY IN SOUTHERN ITALY}

doi:10.1136/jech.2011.142976j.62

${ }^{1} \mathrm{~A}$ Osella, ${ }^{2} \mathrm{M}$ del Pilar Díaz, ${ }^{1} \mathrm{R}$ Cozzolongo, ${ }^{1} \mathrm{~S}$ Elba, ${ }^{1} \mathrm{~J}$ Petruzzi, ${ }^{1} \mathrm{G}$ Leandro, ${ }^{1} G$ Buongiorno, 'V Gianuzzi. 'IRCCS Saverio de Bellis, Castellana Grotte, Bari, Italy; ${ }^{2}$ Escuela de Nutrición, Facultad de Ciencias Médicas, Universidad Nacional de Córdoba, Córdoba, Argentina

Introduction Non-alcoholic fatty liver disease (NAFLD) is the most common liver disease worldwide.

Objectives To estimate NAFLD prevalence and transition probability through different severity stages in a population older than 18 years of age.

Methods The survey was conducted from July 2005 to January 2007 in Putignano, Bari. A systematic random sample of 2301 subjects from the list of records maintained by general practitioners was drawn. Subjects were asked to complete a questionnaire regarding sociodemographic characteristics and life styles, underwent anthropometric measures and liver echography. A sequential logit model was used to estimate the effect of the explanatory variables on the odds and probabilities of passing a set of transitions.

Results Overall prevalence of NAFLD was $24.3 \%$ (male $36.7 \%$, female $14.2 \%$ ); NAFLD steady raised with age. BMI and its interaction with age resulted significantly associated with the odds of transition from less to more severe NAFLD, especially in early stages. At 40 years old the transition probability to pass from no NAFLD to any form of NAFLD reached $35 \%$ and $8 \%$ among men and women respectively. The effect of BMI on the most severe stage 